\title{
Pancreatic Cancer: Clinical Significance of Biomarkers
}

\author{
Kenoki Ohuchida ${ }^{a}$ b Takao Ohtsuka $^{a}$ Kazuhiro Mizumoto ${ }^{a}$ \\ Makoto Hashizume ${ }^{b}$ Masao Tanaka ${ }^{a}$ \\ Departments of a Surgery and Oncology and ${ }^{\mathrm{b}}$ Advanced Medical Initiatives, \\ Graduate School of Medical Sciences, Kyushu University, Fukuoka, Japan
}

\section{Key Message}

Recent advances in our understanding of pancreatic carcinogenesis and the tumor microenvironment have enabled the identification of biomarkers that can be used for screening, diagnosis and prediction of therapeutic response, including the response to novel agents targeting specific cancer cell subpopulations.

\section{Practical Implications}

Although genetic mutations (found in $K$-ras, $p 53$ and $p 16 / N K 4 A$ ) as well as high levels of telomerase activity are frequently associated with pancreatic cancer, their applicability as biomarkers for clinical use has not yet been established. Microarray studies have identified several proteins related to pancreatic cancer. Several of these molecules, such as S100P, MUC1 and MUC5AC, can be quantified in plasma and other body fluids, indicating their utility as potential biomarkers. Research on gemcitabine resistance has uncovered several candidate markers, including genes related to the metabolism and transport of gemcitabine, which may provide a means for identifying those patients who will not benefit from gemcitabine therapy. MicroRNA (miRNA) profiling has revealed an array of miRNAs that are differentially expressed: for example, miR-142-5p and miR-204 may be predictors of response to gemcitabine-based therapy in patients with resected pancreatic cancer. Molecules secreted by pancreatic stellate cells, such as CD10 and CD271, could provide novel targets for the cell-specific management of pancreatic cancer. The use of duodenal juice provides a promising new approach in pancreatic cancer screening. 


\title{
Key Words
}

Biomarker · Pancreatic cancer · Pancreatic stellate cell

\begin{abstract}
Background: Improvement in the prognosis of patients with pancreatic cancer, novel effective screening and diagnostic strategies and treatments are needed. Recent advances in the understanding of pancreatic carcinogenesis and tumor microenvironment have allowed identification of biomarkers for screening, diagnosis and prediction of cancer treatments, including novel therapies targeting specific cancer or stromal cell subpopulations. Personalized therapy in pancreatic cancer is also promising as several drugs such as $\mathrm{S} 1$, capecitabine and gemcitabine reportedly have significant therapeutic effects. Predictive markers are thus needed to select patients most likely to benefit from therapies based on gemcitabine or other drugs. Summary: We review the clinical significance of promising screening, diagnostic, predictive and prognostic biomarkers based on genetic and epigenetic alterations and microRNA abnormalities in pancreatic cancer. We also review new types of biomarkers based on stromal cells, such as pancreatic stellate cells, in the microenvironment of pancreatic cancer.
\end{abstract}

(C) 2013 S. Karger AG, Basel

\section{Introduction}

Pancreatic cancer is the fourth most common cause of tumor-related death in the industrialized world [1]. Only 10-20\% of pancreatic cancer patients are candidates for surgery at the time of presentation, and $<20 \%$ of patients who undergo curative resection are alive after 5 years [2]. Despite recent technological and medical advances, no modalities currently exist for early detection of pancreatic cancer. Aside from a few recent articles describing the successful use of chemotherapy [3], effective treatments for advanced pancreatic cancer, including local and metastatic disease, have not been reported. To improve the prognosis of patients with pancreatic cancer, novel effective screening strategies and treatments are needed.

Intraductal papillary mucinous neoplasm (IPMN) of the pancreas is a distinct entity characterized by massive dilatation of the pancreatic duct, mucin (MUC) hypersecretion and intraductal papillary growth [4]. The histologic spectrum of IPMN is broad, ranging from adenoma to invasive carcinoma. Advances in diagnostic modalities have improved recognition of IPMN in the clinical settings [5]. However, predicting the malignant potential of IPMN before surgical resection is difficult, even if such advanced modalities are used. Therefore, novel diagnostic tools to evaluate the malignant potential of IPMN are needed.

Recent advances in the understanding of pancreatic carcinogenesis and its microenvironment have contributed to the identification of promising biomarkers for screening or diagnosis and the development of novel therapies that target specific subpopulations of cancer cells or stromal cells (fig. 1). Here, we review the clinical significance of biomarkers for pancreatic cancer and IPMN. We also focus on new types of biomarkers based on stromal cells, such as pancreatic stellate cells (PSCs), in the pancreatic cancer microenvironment.

\section{Genetic Alterations as Biomarkers}

Many genetic alterations have been reported in pancreatic cancer and IPMN. Mutations of $K$-ras and $p 53$ are frequently reported as promising biomarker candidates. Inactivation of $p 53$ has been found in $50-75 \%$ of pancreatic cancers [6]; the role of $p 53$ inactivation in 
Fig. 1. Diagram of sampling for biomarkers and clinical specimens. EUS-FNA = Endoscopic ultrasound-guided fine needle aspiration.

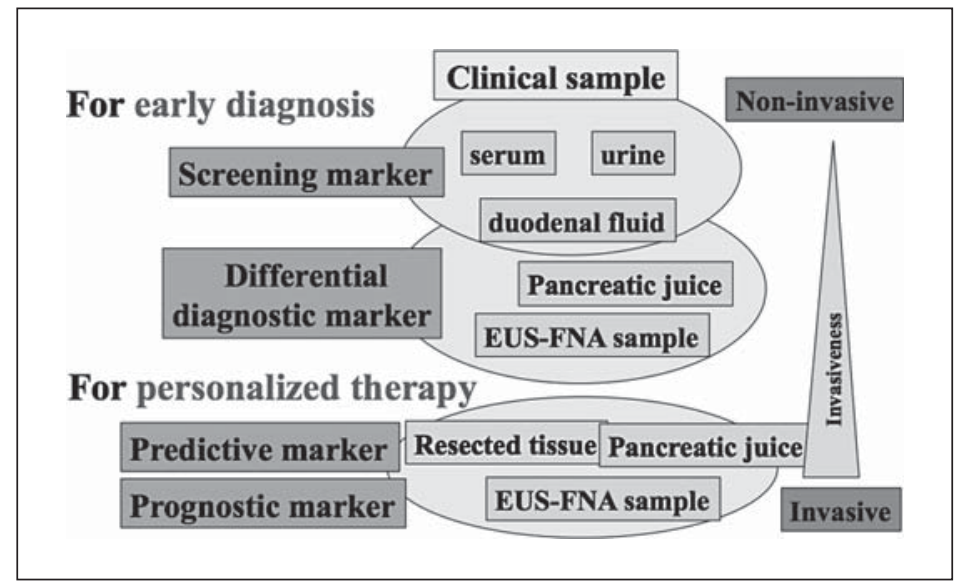

pancreatic carcinogenesis is well known, but its clinical applicability as a biomarker is unclear. Mutation of tumor suppressor genes, such as $p 53$, occurs at numerous hot spots, which could make detection of such mutations too complex and time-consuming for practical clinical use. On the other hand, detection of $K$-ras mutations has been used in a large clinical study, as $K$-ras mutation occurs at a specific codon (codon 12 or 13) [7]. Several researchers performed the analyses using pancreatic juice; however, this method is reported to have detected $K$-ras mutation in 38.1-90\% of patients [7, 8]. These conflicting data show the difficulty of detecting DNA mutations in clinical practice.

The mutation, deletion or promoter hypermethylation of $p 16^{I N K 4 A}$ was found in $80-95 \%$ of pancreatic cancers. The effect of $p 16^{I N K 4 A}$ loss in pancreatic cancer is as well-established as losses of $p 53$ and mutation of $K$-ras. Such $p 16$ alterations are seen in early-stage pancreatic carcinogenesis, such as pancreatic intraepithelial neoplasia (PanIN) and IPMN [9]. K-ras mutations are also seen in early pancreatic carcinogenesis, including PanIN and IPMN processes [9]. Therefore, detecting such mutations in connection with early pancreatic carcinogenesis would be a promising screening tool. However, developing a practical assay to detect mutations or quantify methylation using blood or plasma would be difficult as few such mutations are present in blood or plasma. Reportedly, genomic alterations of SMAD4 (DPC4), LKB1 (STK11) and BRCA2 are found in <50, 4-6 and 17\% of pancreatic cancers, respectively [10]; their clinical significance remains unknown.

\section{Telomerase Activity and Human Telomerase Reverse Transcriptase Expression as Biomarkers}

Telomerase is an RNA-dependent DNA polymerase and is generally inactivated in normal mature cells. In pancreatic carcinogenesis, telomerase is activated to avoid telomere shortening in tumor cells. Pancreatic cancer cells show high levels of telomerase activity, whereas premalignant lesions, such as PanIN lesions and non-malignant IPMN lesions, only show low levels of telomerase activity. Therefore, telomerase activity is a promising definite or differential marker for pancreatic cancer [11]. We and other investigators have reported on the detection of telomerase activity in pancreatic juice in diagnosing pancreatic cancer [12]. However, clinical use of this marker for cancer diagnosis is currently unfeasible because of the impracticality of evaluating sample quality and because of the difficulties of quantitative measurement. Human telomerase reverse transcriptase (hTERT) is a telomerase subunit, 
and its mRNA has been suggested as a diagnostic marker. We performed a large-scale analysis of 88 samples of pancreatic juice that included quantitative analyses of $h T E R T$ mRNA to differentiate pancreatic cancer from IPMN or chronic pancreatitis [13]. The hTERT mRNA levels were significantly greater in pancreatic cancer-derived juice samples than in IPMNderived samples, which suggested that pancreatic juice analyses based on $h T E R T$ expression could discriminate between pancreatic cancer and non-malignant IPMN. However, the data also indicated that hTERT expression analyses were not suitable for differentiating between pancreatic cancer and chronic pancreatitis because lymphocytes isolated from some resected tissues with histologic appearance of pancreatitis also showed relatively high levels of $h T E R T$ mRNA expression.

\section{Cancer-Related Molecules as Biomarkers}

Microarray analysis is a powerful tool for identifying molecules associated with pancreatic cancer. Microarray data recently revealed that expression of members of the S100 and MUC families increases in pancreatic cancer $[14,15]$. Also, many cancer-related molecules, such as growth factors, cytokines and cancer-stromal interaction-related molecules, are reported as prognostic or predictive markers for the treatment of pancreatic cancer.

\section{S100 Family}

S100 family proteins are small $\mathrm{Ca}^{2+}$-binding EF-hand-type proteins that affect regulation of several intra- and extracellular processes, including cell proliferation, differentiation and intracellular signaling. Arumugam et al. [16] reported that S100P expression affects cell growth and invasion in pancreatic cancer. We also found that invasive ductal carcinoma cells, PanIN cells and IPMN cells expressed significantly more S100P than did normal ductal cells $[17,18]$, which suggests that S100P expression is up-regulated in early pancreatic carcinogenesis. We and other investigators also reported on other S100 proteins - S100A6, S100A2 and S100A11 - and found S100A6 and S100A11 to be up-regulated during early-phase pancreatic carcinogenesis [19-22]; quantification of these proteins may help screen or detect pancreatic cancer, whereas S100A2 was a prognostic marker in pancreatic cancer.

Although analysis of S100 proteins in pancreatic juice may have limited utility in discriminating pancreatic cancer from benign neoplasms, it may allow more effective screening of patients with high-risk lesions. Several S100 family molecules, such as S100P, are secreted and measurable from plasma and other body fluids, suggesting that such molecules are potential screening biomarkers.

\section{MUC Family}

MUCs are high-molecular-weight glycoproteins. In pancreatic cancer, overexpression of MUC1 and MUC6, and de novo expression of MUC4 and MUC5AC, have been observed at both the mRNA and protein levels [23]. DNA microarray technology has shown that MUC4 and MUC5AC are up-regulated in pancreatic cancer [15]. We also reported that MUC1 and MUC5AC mRNA levels were significantly increased in pancreatic cancer and in pancreatic juice from patients with pancreatic cancer [24]. ROC curve analyses of MUC1 and MUC5AC expression revealed the usefulness of quantifying these two markers for the diagnosis of pancreatic cancer. These data suggest that quantitative analysis of MUC1 and MUC5AC levels in pancreatic juice offers a definitive preoperative diagnosis of pancreatic cancer and possible detection of early pancreatic cancer. 


\section{Function-Related Molecules and Personalized Therapy}

Many function-related factors, such as epidermal growth factor, insulin-like growth factor, hepatocyte growth factor, fibroblast growth factor, transforming growth factor-1, VEGF, MMP2, MMP7, MMP9, hENT1, Sonic hedgehog and their related receptors, have been reported as potential prognostic or predictive markers for patient survival or therapeutic efficacy in treating pancreatic cancer. Personalized therapies are increasingly used for several types of cancers, such as breast cancer, lung cancer, colon cancer and gastric cancer. Personalized therapy in pancreatic cancer is also promising and the establishment of such a therapy is needed. Gemcitabine is widely accepted as a first-line treatment for patients with advanced or resected pancreatic cancer. However, in advanced pancreatic cancer, the complete plus partial response rate and the disease control rate have been shown to be 8.0-13.5 and 49.2-62.1\%, respectively, even with combination treatment arms [3]; this implies that many patients with pancreatic cancer do not benefit from gemcitabine-based combination therapies. Predictive markers are thus needed to select patients most likely to benefit from therapies based on gemcitabine or other drugs. Research on gemcitabine resistance has identified several candidate markers, including genes related to gemcitabine metabolism and transport, such as those for deoxycytidine kinase, ribonucleotide reductase and human equilibrative nucleoside transporter-1 [25].

Recently, several drugs, such as S1 and capecitabine, were reported to have significant therapeutic effects as effective as gemcitabine in treating pancreatic cancer. Personalized therapy based on predictive markers could provide a more appropriate treatment for specific subsets of patients with pancreatic cancer.

\section{MicroRNAs as Biomarkers}

MicroRNAs (miRNAs) are small noncoding RNA gene products of approximately 22 nucleotides that are found in a variety of organisms. Although the biologic functions of most miRNAs are not yet fully understood, they are apparently involved in various biologic processes, including cell proliferation, cell death, stress resistance and fat metabolism, through regulation of gene expression [26].

Increasing evidence indicates that miRNAs are mutated or differentially expressed in many cancer types. Especially, miR-21, miR-196a-2, miR-155, and miR-210 in pancreatic cancer significantly correlate with poor prognosis [27-29]. Furthermore, microarray-based miRNA profiles have identified miRNAs that are differentially expressed in pancreatic cancer [27]. We also performed microarray-based miRNA profiling and identified 24 candidate miRNAs that were up- or down-regulated in gemcitabine-resistant cells [30]. Our results showed that patients with 'high' miR-142-5p and miR-204 expression had significantly longer survival than those with 'low' miR-142-5p and miR-204 expression in the gemcitabine-treated group, but not in the non-gemcitabine-treated group; these data suggest that miR-142-5p and miR-204 predict chemotherapeutic responses in patients with resected pancreatic cancer.

\section{Stromal Biomarkers Based on Pancreatic Cancer Microenvironment}

Pancreatic cancer is characterized by excessive desmoplasia, in which extracellular matrix and the number of cells that express $\alpha$-smooth muscle actin increase [31]. Desmoplasia contributes to aggressiveness in pancreatic cancer and to resistance against traditional therapies through tumor-stromal interactions [32]. Recently, PSCs have been identified as the principal source of the excessive matrix in chronic pancreatitis and pancreatic adenocarcinoma [33]. Soluble factors secreted by activated PSCs also promote malignant phenotypes and resis- 
Fig. 2. Cell-specific biomarkers and personalized therapy for pancreatic cancer.

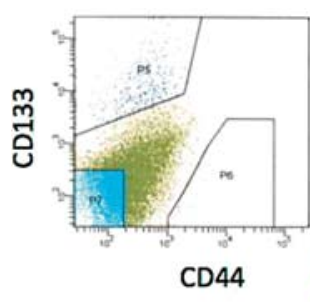

CD10/aSMA

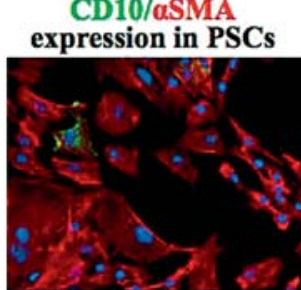

\section{Cancer stem cell}
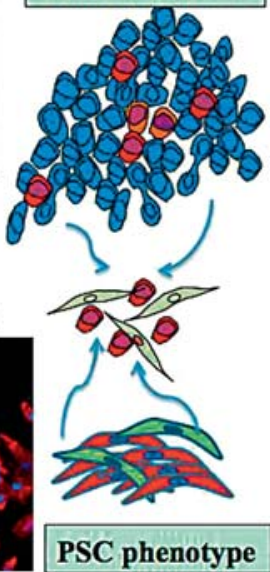

tance to gemcitabine or radiation therapy in pancreatic cancer cells [34]. Previous research focused on the role of cancer stem cells in various cancers; cancer stem cells comprise a very small population of cancer cells that can initiate and sustain tumor formation [35], whereas myofibroblasts and mesenchymal cells isolated from various human tissues, including PSCs, exhibit similar phenotypes based on their cell surface markers. However, specific PSC phenotypes with different functions remain unknown. Recently, we focused on such PSC heterogeneity and found, using in vitro and in vivo experiments, that PSCs have functional heterogeneity similar to cancer stem cells; PSCs that express CD10 (a 90-110-kDa zinc-dependent cell surface metalloproteinase) are the leading cell population in promoting pancreatic cancer [36]. We also found, using immunohistochemical analyses, that stromal CD10 expression in PSCs is significantly associated with poor prognosis in pancreatic cancer. These data suggest that CD10 expression in PSCs is a prognostic predictor for pancreatic cancer patients, and that CD10+ PSCs are a promising target for novel therapies to control desmoplasia.

We also reported on another PSC phenotype, based on CD271 expression [37]. CD271 (nerve growth factor receptor or p75NTR) is a neurotrophin receptor that has been implicated in paracrine growth regulation of several neuronal and non-neuronal tumor types [38]. In the pancreas, CD271 expression in PSCs has been detected [39]; CD271+ PSCs were frequently observed on the periphery, rather than the center, of pancreatic tumors. High stromal CD271 expression was associated with a good prognosis $(p=0.0040)$ [37]. We also found that stromal cells were strongly stained for CD271 around PanIN and IPMN lesions. These data imply that CD271+ PSCs appear at early-stage pancreatic carcinogenesis, and CD271+ PSCs have different roles from CD10+ PSCs, such as defense against cancer. Findings based on stromal phenotypes in the pancreatic cancer microenvironment may lead to the discovery of new biomarkers for cell-specific management of pancreatic cancer (fig. 2).

\section{Conclusion}

Identifying biomarkers for the diagnosis of pancreatic cancer is more difficult than identifying its prognostic or predictive markers because of the use of small samples obtained preoperatively, such as pancreatic juice or fine needle aspiration samples. Identification of 
screening biomarkers is also much more difficult because of the use of blood, serum or stool. Recently, we have been investigating analysis of duodenal juices to establish a new screening method. Duodenal juice is collected more easily than pancreatic juice because it is not necessary to insert a cannula into the pancreatic duct, and it more directly contains materials related to pancreatic lesions than do blood, serum or stool. Therefore, duodenal juice is a promising modality for pancreatic cancer screening.

Several genetically engineered mouse models have been developed for pancreatic cancer which facilitate the study of the pancreatic microenvironment. Tumors in these models cause desmoplasia similar to that seen in human pancreatic cancer. These models also enable analyses of pancreatic carcinogens because of similarities between murine and human PanIN.

We hope that ongoing projects will provide novel findings regarding biomarkers associated with pancreatic carcinogenesis and its microenvironment.

\section{Acknowledgement}

Supported in part by a Grant-in-Aid from the Ministry of Education, Culture, Sports, Science and Technology of Japan.

\section{Disclosure Statement}

The authors declare no conflict of interest.

\section{References}

1 Jemal A, Siegel R, Ward E, Murray T, Xu J, Thun MJ: Cancer statistics, 2007. CA Cancer J Clin 2007;57:43-66.

-2 Matsuno S, Egawa S, Fukuyama S, Motoi F, Sunamura M, Isaji S, et al: Pancreatic Cancer Registry in Japan: 20 years of experience. Pancreas 2004;28:219-230.

-3 Ueno H, Ioka T, Ikeda M, Ohkawa S, Yanagimoto H, Boku N, et al: Randomized phase III study of gemcitabine plus S-1, S-1 alone, or gemcitabine alone in patients with locally advanced and metastatic pancreatic cancer in Japan and Taiwan: GEST study. J Clin Oncol 2013;31:1640-1648.

4 Tanaka M, Chari S, Adsay V, Fernandez-del Castillo C, Falconi M, Shimizu M, et al: International consensus guidelines for management of intraductal papillary mucinous neoplasms and mucinous cystic neoplasms of the pancreas. Pancreatology 2006;6:17-32.

5 Miyasaka Y, Nagai E, Yamaguchi H, Fujii K, Inoue T, Ohuchida K, et al: The role of the DNA damage checkpoint pathway in intraductal papillary mucinous neoplasms of the pancreas. Clin Cancer Res 2007;13:4371-4377.

-6 Redston MS, Caldas C, Seymour AB, Hruban RH, da Costa L, Yeo CJ, et al: p53 mutations in pancreatic carcinoma and evidence of common involvement of homocopolymer tracts in DNA microdeletions. Cancer Res 1994;54: 3025-3033.

7 Trumper L, Menges M, Daus H, Kohler D, Reinhard JO, Sackmann M, et al: Low sensitivity of the ki-ras polymerase chain reaction for diagnosing pancreatic cancer from pancreatic juice and bile: a multicenter prospective trial. J Clin Oncol 2002;20:4331-4337.

8 Tada M, Omata M, Kawai S, Saisho H, Ohto M, Saiki RK, et al: Detection of ras gene mutations in pancreatic juice and peripheral blood of patients with pancreatic adenocarcinoma. Cancer Res 1993;53:2472-2474.

-9 Delpu Y, Hanoun N, Lulka H, Sicard F, Selves J, Buscail L, et al: Genetic and epigenetic alterations in pancreatic carcinogenesis. Curr Genomics 2011;12:15-24.

10 Murphy KM, Brune KA, Griffin C, Sollenberger JE, Petersen GM, Bansal R, et al: Evaluation of candidate genes MAP2K4, MADH4, ACVR1B, and BRCA2 in familial pancreatic cancer: deleterious BRCA2 mutations in $17 \%$. Cancer Res 2002;62:3789-3793.

11 Suehara N, Mizumoto K, Muta T, Tominaga Y, Shimura H, Kitajima S, et al: Telomerase elevation in pancreatic ductal carcinoma compared to nonmalignant pathological states. Clin Cancer Res 1997;3:993-998.

12 Myung SJ, Kim MH, Kim YS, Kim HJ, Park ET, Yoo KS, et al: Telomerase activity in pure pancreatic juice for the diagnosis of pancreatic cancer may be complementary to K-ras mutation. Gastrointest Endosc 2000;51:708713.

13 Ohuchida K, Mizumoto K, Yamada D, Yamaguchi H, Konomi H, Nagai E, et al: Quantitative analysis of human telomerase reverse transcriptase in pancreatic cancer. Clin Cancer Res 2006;12:2066-2069. 
Crnogorac-Jurcevic T, Missiaglia E, Blaveri E, Gangeswaran R, Jones M, Terris B, et al: Molecular alterations in pancreatic carcinoma: expression profiling shows that dysregulated expression of S100 genes is highly prevalent. J Pathol 2003;201:63-74.

15 Iacobuzio-Donahue CA, Ashfaq R, Maitra A, Adsay NV, Shen-Ong GL, Berg K, et al: Highly expressed genes in pancreatic ductal adenocarcinomas: a comprehensive characterization and comparison of the transcription profiles obtained from three major technologies. Cancer Res 2003;63:8614-8622.

16 Arumugam T, Simeone DM, Van Golen K, Logsdon CD: S100P promotes pancreatic cancer growth, survival, and invasion. Clin Cancer Res 2005;11:5356-5364.

17 Ohuchida K, Mizumoto K, Egami T, Yamaguchi H, Fujii K, Konomi H, et al: S100P is an early developmental marker of pancreatic carcinogenesis. Clin Cancer Res 2006;12:5411-5416.

18 Nakata K, Nagai E, Ohuchida K, Hayashi A, Miyasaka Y, Aishima S, et al: S100P is a novel marker to identify intraductal papillary mucinous neoplasms. Hum Pathol 2010;41:824-831.

19 Biankin AV, Kench JG, Colvin EK, Segara D, Scarlett CJ, Nguyen NQ, et al: Expression of S100A2 calcium-binding protein predicts response to pancreatectomy for pancreatic cancer. Gastroenterology 2009;137:558-568, 568.e1-e11.

20 Ohuchida K, Mizumoto K, Ohhashi S, Yamaguchi H, Konomi H, Nagai E, et al: S100A11, a putative tumor suppressor gene, is overexpressed in pancreatic carcinogenesis. Clin Cancer Res 2006;12:5417-5422.

-21 Ohuchida K, Mizumoto K, Yu J, Yamaguchi H, Konomi H, Nagai E, et al: S100A6 is increased in a stepwise manner during pancreatic carcinogenesis: clinical value of expression analysis in 98 pancreatic juice samples. Cancer Epidemiol Biomarkers Prev 2007;16:649-654.

22 Ohuchida K, Mizumoto K, Miyasaka Y, Yu J, Cui L, Yamaguchi H, et al: Over-expression of S100A2 in pancreatic cancer correlates with progression and poor prognosis. J Pathol 2007;213:275-282.

23 Kim GE, Bae HI, Park HU, Kuan SF, Crawley SC, Ho JJ, et al: Aberrant expression of MUC5AC and MUC6 gastric mucins and sialyl Tn antigen in intraepithelial neoplasms of the pancreas. Gastroenterology 2002;123:10521060.

24 Ohuchida K, Mizumoto K, Yamada D, Fujii K, Ishikawa N, Konomi H, et al: Quantitative analysis of MUC1 and MUC5AC mRNA in pancreatic juice for preoperative diagnosis of pancreatic cancer. Int J Cancer 2006; 118: 405-411.

25 Farrell JJ, Elsaleh H, Garcia M, Lai R, Ammar A, Regine WF, et al: Human equilibrative nucleoside transporter 1 levels predict response to gemcitabine in patients with pancreatic cancer. Gastroenterology 2009;136: 187-195.

26 Ambros V: MicroRNA pathways in flies and worms: growth, death, fat, stress, and timing. Cell 2003;113:673676.

27 Bloomston M, Frankel WL, Petrocca F, Volinia S, Alder H, Hagan JP, et al: MicroRNA expression patterns to differentiate pancreatic adenocarcinoma from normal pancreas and chronic pancreatitis. JAMA 2007;297: 1901-1908.

-28 Greither T, Grochola LF, Udelnow A, Lautenschläger C, Würl P, Taubert H: Elevated expression of microRNAs 155, 203, 210 and 222 in pancreatic tumors is associated with poorer survival. Int J Cancer 2010;126:73-80.

29 Dillhoff M, Liu J, Frankel W, Croce C, Bloomston M: MicroRNA-21 is overexpressed in pancreatic cancer and a potential predictor of survival. J Gastrointest Surg 2008;12:2171-2176.

-30 Ohuchida K, Mizumoto K, Kayashima T, Fujita H, Moriyama T, Ohtsuka T, et al: MicroRNA expression as a predictive marker for gemcitabine response after surgical resection of pancreatic cancer. Ann Surg Oncol 2011;18:2381-2387.

-31 Omary MB, Lugea A, Lowe AW, Pandol SJ: The pancreatic stellate cell: a star on the rise in pancreatic diseases. J Clin Invest 2007;117:50-59.

-32 Bachem MG, Zhou S, Buck K, Schneiderhan W, Siech M: Pancreatic stellate cells - role in pancreas cancer. Langenbecks Arch Surg 2008;393:891-900.

-33 Apte MV, Park S, Phillips PA, Santucci N, Goldstein D, Kumar RK, et al: Desmoplastic reaction in pancreatic cancer: role of pancreatic stellate cells. Pancreas 2004;29:179-187.

-34 Hwang RF, Moore T, Arumugam T, Ramachandran V, Amos KD, Rivera A, et al: Cancer-associated stromal fibroblasts promote pancreatic tumor progression. Cancer Res 2008;68:918-926.

35 Li C, Heidt DG, Dalerba P, Burant CF, Zhang L, Adsay V, et al: Identification of pancreatic cancer stem cells. Cancer Res 2007;67:1030-1037.

36 Ikenaga N, Ohuchida K, Mizumoto K, Cui L, Kayashima T, Morimatsu K, et al: CD10+ pancreatic stellate cells enhance the progression of pancreatic cancer. Gastroenterology 2010;139:1041-1051, 1051.e1-e8.

-37 Fujiwara K, Ohuchida K, Mizumoto K, Shindo K, Eguchi D, Kozono S, et al: CD271(+) subpopulation of pancreatic stellate cells correlates with prognosis of pancreatic cancer and is regulated by interaction with cancer cells. PLoS One 2012; 7:e52682.

38 Micera A, Lambiase A, Stampachiacchiere B, Bonini S, Levi-Schaffer F: Nerve growth factor and tissue repair remodeling: trkA(NGFR) and p75(NTR), two receptors one fate. Cytokine Growth Factor Rev 2007;18:245256.

-39 Trim N, Morgan S, Evans M, Issa R, Fine D, Afford S, et al: Hepatic stellate cells express the low affinity nerve growth factor receptor p75 and undergo apoptosis in response to nerve growth factor stimulation. Am J Pathol 2000;156:1235-1243. 